\title{
Interférences
}

Ars scribendi

$9 \mid 2016$

Varia

\section{Il ritratto del bellus homo in Mart. 3, 63: realismo e retorica}

Francesco Berardi

\section{(2) OpenEdition}

1 Journals

\section{Edizione digitale}

URL: http://journals.openedition.org/interferences/5925

DOI: $10.4000 /$ interferences.5925

ISSN: $1777-5485$

\section{Editore}

HiSoMA - Histoire et sources des Mondes antiques

\section{Notizia bibliografica digitale}

Francesco Berardi, « II ritratto del bellus homo in Mart. 3, 63: realismo e retorica », Interférences [En ligne], 9 | 2016, mis en ligne le 18 janvier 2018, consulté le 15 septembre 2020. URL : http:// journals.openedition.org/interferences/5925; DOI : https://doi.org/10.4000/interferences.5925

Questo documento è stato generato automaticamente il 15 settembre 2020.

Tous droits réservés 


\title{
Il ritratto del bellus homo in Mart. 3, 63: realismo e retorica
}

\author{
Francesco Berardi
}

\author{
Non hic Centauros, non Gorgonas Harpyiasque \\ invenies: hominem pagina nostra sapit \\ «non troverai qui i Centauri, non le Gorgoni o le \\ Arpie: \\ la mia pagina sa di uomo» (Mart. 10, 4, 9-10).
}

1 Con questo celebre distico Marziale, poeta latino d'età imperiale (40-110 d.C.), nato nella Spagna Tarragonese, ben presto trapiantato a Roma, rivendica orgogliosamente il rifiuto di praticare i toni solenni ed impegnativi dell'epica, qui simboleggiata dalle mostruose figure delle Gorgoni e delle Arpie, a favore di una poesia più dimessa, vicina al quotidiano scorrere delle giornate, alle tematiche umili della vita comune, la quale deve trasparire, assaporarsi (sapere), attraverso i brevi, ma densi versi dell'epigramma. Aderendo ad un gusto e ad una tradizione poetica inaugurata a Roma da Catullo, anche Marziale sceglie le nugae spiritose, suggerite da una Musa prepotente. Lo scopo del suo poetare è la rappresentazione vivida e divertente di un'umanità colta nei suoi difetti e nelle sue piccolezze. Protagonista dei suoi epigrammi è, infatti, la Roma dei Flavii, una società dall'esistenza tumultuosa e caotica, popolata da personaggi ridicoli e miserabili. Contro questi Marziale non usa mai il tono moralistico della condanna, né l'atteggiamento di una satira aggressiva, ma la sanzione del riso, una comicità leggera che, più che insegnare o biasimare, vuole solo offrire un'immagine della vita comune e dei costumi della gente: una sorta di specchio dinanzi al quale i Romani possono guardare per riflettere su loro stessi e sul proprio modo di vivere. Per questo egli usa le maschere: il ricorso a figure tipiche serve a irrigidire nella fissità e nell'anonimato del personaggio quelle situazioni e quei difetti che l'autore ha deciso di riprodurre nella finzione poetica, sfumando ogni possibile tono aggressivo. L'aspirazione è allora rappresentare la vita con immediatezza in modo da restituirne un'immagine divertente, capace di suscitare riflessioni ben più profonde sulla condizione morale degli uomini del tempo ${ }^{1}$. 
2 Per tali corde si è parlato a ragione di realismo, perché negli epigrammi di Marziale ognuno può riconoscere gli atteggiamenti, i gesti e i vizi di chi gli sta accanto, ritrovare le situazioni della vita vera, rivivere le scene di ogni giorno con trasporto e sincera partecipazione, così da leggere la poesia ed esclamare soddisfatti: "è cosa mia!"2 . E benché spesso nella breve e preziosa dimensione dell'epigramma affiori un'estrema cura formale, fatta di giochi di chiasmi, parallelismi, metafore e figure di ogni specie, la retorica, troppo spesso confusa con la pedante stilistica, è stata considerata aliena da questa poesia. Scrisse Concetto Marchesi: “Arte realistica non è già quella che si fa serva e nomenclatrice delle cose, ripetendone i nomi e le forme, bensì quella che le cose compone e risuscita in modo che esse abbiano vigore e potenza nello spirito nostro. Ed è questa l'arte di Marziale che, nei secoli della retorica stucchevole e imbellettata, pare a chi ben la osservi e la consideri, un prodigio"3.

3 Sul commento del noto latinista grava il pregiudizio che vuole la retorica mero artificio e, in quanto tale, estranea ad ogni forma di poesia che intenda proporre un rapporto diretto, realistico, con la vita quotidiana ${ }^{4}$. Ma questa è una vecchia idea di retorica, un'idea romantica, ormai superata dalle odierne riflessioni sul fare poetico e letterario degli antichi, in cui appare chiaro che gli studi retorici costituivano parte fondamentale del bagaglio di competenze linguistiche e culturali dello scrittore, influenzando notevolmente le forme e le modalità della letteratura ${ }^{5}$.

4 Marziale, e come lui tutti gli altri scrittori latini almeno a partire dal I sec. a.C., si formano attraverso un lungo percorso di exercitatio retorica in cui hanno affinato le loro naturali doti di scrittura ${ }^{6}$. Dopo aver appreso alla scuola del grammatico le nozioni di base per esprimersi in una lingua corretta, grazie ai primi studi retorici gli scrittori antichi imparano le tecniche di esposizione, i meccanismi argomentativi e i procedimenti espressivi necessari per scrivere in modo chiaro e brillante, costruendo una storia credibile, un mondo letterario finto, ma verosimile, che rapisca il lettore/ ascoltatore con la forza coinvolgente delle emozioni. Queste competenze vengono assicurate attraverso una serie graduata di esercizi scolastici, i progymnasmata, che consistono nella composizione di alcune tipologie di testo considerate fondamentali per l'apprendimento della bella scrittura, come la favola, il racconto, l'aneddoto, il luogo comune, l'encomio, la descrizione, l'etopea ${ }^{7}$. Si tratta di un'autentica palestra di scrittura che, ricorda Teone, contemporaneo forse di Marziale, non è solo utile al futuro oratore, ma anche ad ogni letterato, poeta e storiografo che sia ${ }^{8}$. Durante questi studi i ragazzi apprendono le tecniche espositive, leggendo i classici e rielaborandone i modelli narrativi e stilistici per arrivare a testi personali ed originali.

Benché non ci siano pervenuti manuali in lingua latina, ma solo la trattazione di Quintiliano in inst. 2, 4 e rapidi accenni nel de grammaticis et rhetoribus di Svetonio ${ }^{9}$, siamo sufficientemente informati che questa è la prassi didattica diffusa a Roma almeno dal I sec. a.C. e che a Roma questa prassi è giunta dalla Grecia ove il curriculum di studi si è assemblato a partire dall'età ellenistica ${ }^{10}$. I progymnasmata sono strumento di allenamento alla scrittura, praticato sia in età scolastica, sia in età adulta, come mostra l'esperienza di Cicerone prima, di Quintiliano poi, il quale raccomanda di non lasciare mai inaridire la propria vena compositiva praticando continuamente questi esercizi, anche dopo gli studi superiori, quando cioè l'oratore si è ormai formato grazie allo studio delle più complesse tecniche di argomentazione e delle più elaborate figure stilistiche ed è ormai capace di comporre i discorsi fittizi (le declamazioni) ${ }^{11}$. 
6 Marziale ebbe un'educazione del genere, anzi un'ottima formazione retorica, se sono vere le notizie secondo le quali frequentò la scuola retorica di Bilbilis, obbedendo al desiderio dei genitori che sognavano per lui la carriera forense.

Non sarà dunque fuori luogo applicare anche a lui, come già è stato proficuamente fatto per altri autori ${ }^{12}$, la lente dell'analisi retorica per individuare luoghi della sua poesia in cui affiorino forme espressive e procedimenti narrativi magari ereditati dalla tecnografia antica, appresi nella scuola di Bilbilis e ora per sempre fissati nella struttura narrativa e nella veste stilistica dei suoi celebri epigrammi. Ma più che soffermarci sulla mera indagine stilistica, sulla semplice osservazione delle figure dell'elocuzione (metafore, tropi, figure di pensiero e di parola), che in Marziale sono tante e costituiscono il frutto di un diuturno labor limae, noi vogliamo piuttosto individuare quelli che possono essere definiti "codici retorici"; intendiamo, cioè, vedere se esistono modelli di riferimento tanto nello stile quanto nella composizione testuale (scelta e organizzazione degli argomenti) che sono ripresi dalla retorica e rielaborati dal poeta persino in un epigramma apparentemente così distante dall'artificiosa costruzione della retorica. È una sorta di sfida, perché intendiamo verificare se la retorica, con i suoi principi normativi, con i suoi canoni di composizione e di scrittura, costituisca talora l'ossatura stessa di questa poesia vivida che "sa di uomo". E possiamo anticipare le conclusioni: questa sfida è vinta. Negli epigrammi di Marziale non solo troviamo l'applicazione di codici retorici, ma anche constatiamo che talvolta i procedimenti espositivi definiti dai retori costituiscono l'elemento fondamentale su cui è strutturata la poesia, persino la chiave interpretativa per capirne il realismo e il garbato umorismo. La retorica diviene allora strumento di esegesi capace di attribuire nuovi significati a testi già ampiamente commentati e in grado di spiegare come e perché i versi di Marziale divengono immagine del reale.

Per esemplificare questo approccio critico al testo, ho selezionato un epigramma della raccolta di Marziale, il 63 del terzo libro, perché può considerarsi in qualche modo paradigmatico delle forme stilistiche usate da Marziale e dell'indagine retorica ad esse collegata. Nella galleria dei personaggi che popolano questa Roma vacua e caotica, fatta di avari, parassiti, ubriaconi, ladri, poetastri, medici incapaci e vecchie orribili, non può mancare il tipo umano elegante e vanitoso, il dandy che cura il suo corpo, veste alla moda, affetta raffinatezza e frequenta i salotti mondani. È l'emblema di una società falsa e frivola che ostenta eleganza esteriore nella misura in cui è vuota di principi morali. Ma non esiste la condanna, esiste solo il riso di Marziale, che si appunta su questo personaggio in cerca di autore, ritratto come tanti altri nel ridicolo dei suoi comportamenti.

Cotile, bellus homo es: dicunt hoc, Cotile, multi. Audio: sed quid sit, dic mihi, bellus homo.

"Bellus homo est, flexos qui digerit ordine crines, balsama qui semper, cinnama semper olet; cantica qui Nili, qui Gaditana susurrat, qui movet in varios bracchia volsa modos; inter femineas tota qui luce cathedras desidet atque aliqua semper in aure sonat, qui legit hinc illinc missas scribitque tabellas; pallia vicini qui refugit cubiti; qui scit, quam quis amet, qui per convivia currit, Hirpini veteres qui bene novit avos". 
Quid narras? hoc est, hoc est homo, Cotile, bellus?

Res pertricosa est, Cotile, bellus homo.

" 'Cotilo, sei un uomo elegante: lo dicono molti, Cotilo.

Lo sento dire: ma dimmi, chi è l'uomo elegante?

"L'uomo elegante è colui che dispone con arte i capelli arricciati,

che odora sempre di balsamo, odora sempre di cinnamomo,

che sussurra canti del Nilo e di Cadice,

che muove le braccia depilate secondo ritmi diversi;

che siede per tutto il giorno tra le poltrone delle donne

e sempre bisbiglia loro qualcosa nell'orecchio;

che legge e scrive bigliettini mandati qua e là;

che si tiene lontano dal mantello di chi mangia vicino a lui,

che sa chi ami chi, che corre da un banchetto all'altro,

che conosce i vecchi antenati del cavallo Irpino".

Che cosa descrivi? È questo, è questo, Cotilo, un uomo elegante?

Un uomo elegante è ... una cosa troppo complicata'. »

L'epigramma è concepito come un breve dialogo tra il protagonista, Cotilo, nome parlante che già rivela il tipo umano chiacchierone, seducente, tutto moine $\mathrm{e}$ smancerie $e^{13}$, e il poeta osservatore. Il dialogo muove da una constatazione: Cotilo è un bellus homo, come dicono molti, e dalla richiesta di definizione da parte del poeta: che cos'è un bellus homo? Si noti: non chi, ma che cos'è (quid) il bellus homo. Segue quindi la risposta del personaggio, che non offre una definizione analitica del tipo umano, ma si dilunga in un'accurata descrizione dei suoi comportamenti caratteristici, suscitando, suo malgrado, il riso ad ogni ulteriore pennellata: il bellus homo si pettina i capelli, profuma di balsamo e cinnamomo, canticchia canzoni egiziane e spagnole, danza muovendo le sue braccia depilate, siede tra le signore bisbigliando all'orecchio pettegolezzi, manda e riceve bigliettini, si discosta schizzinoso dal mantello del vicino, è informato sugli amori e sui temi dell'attualità mondana. Alla fine della lunga descrizione del personaggio il poeta non può che rispondere, tirando lui stesso le conclusioni: il bellus homo è ... una cosa molto complicata!

Davvero potente è l'hapax, pertricosa, che condensa nella novità della parola il forte effetto provocato dalla battuta a sorpresa ${ }^{14}$. L'attesa di una definizione seria viene delusa a favore di una conclusione ironica, ma non per questo meno illuminante o meno veritiera della reale essenza del personaggio, grazie alla notevole capacità espressiva dell'aprosdoketon.

11 Come già annunciato, l'epigramma può considerarsi emblematico della produzione poetica di Marziale. In esso ritroviamo tutte le caratteristiche di tecnica narrativa e di stile che i filologi e gli studiosi della letteratura hanno apprezzato. Possiamo ad esempio rinvenire, come fece per primo il Lessing, una tensione tra il momento descrittivo, particolarmente esteso, detto Erwartung, che ha il compito di creare e prolungare l'attesa, e la rapida battuta finale, Aufschluss, che la scioglie ${ }^{15}$. Oppure possiamo seguire l'analisi di Kruuse e notare la netta bipartizione dell'epigramma in una proposizione (domanda) e in una risposta conclusiva, che contiene la pointe $e^{16}$. Ritroviamo poi la tecnica dell'accumulo: nella prima parte dell'epigramma il poeta riunisce in sequenza immagini di un carattere e di un comportamento, costruendo un repertorio brillante di situazioni simili, per poi illuminare con immediatezza l'insieme dei fatti grazie al fulmen in clausula ${ }^{17}$. Oppure apprezziamo il tono sentenzioso che caratterizza la battuta finale, dove nelle forme espressive di una massima di valore universale è racchiuso l'intervento del tutto soggettivo e personale del poeta, che conclude una più o meno estesa sezione precedente costituita dalla descrizione 
oggettiva di un personaggio o di una situazione (come rilevava uno studioso di retorica, il Bartwick) ${ }^{18}$.

Tutto questo c'è nell'epigramma del bellus homo, così come c'è l'ironia che si appunta sulla maschera di un nome fittizio, c'è la rielaborazione di motivi e personaggi topici (di belli homines ne è piena la letteratura latina!) ${ }^{19}$, c'è la denuncia di una società falsa $\mathrm{e}$ cortese, c'è la notevole cura formale, che si manifesta nel sapiente gioco di chiasmi, anafore e parallelismi sintattici ${ }^{20}$, c'è il gusto per la rappresentazione realistica, c'è l'occhio attento a cogliere i comportamenti quotidiani dell'uomo nei suoi vizi e nei suoi difetti e, infine, c'è lo stilus capace di raffigurarli nei versi con la vivezza dei colori e la precisione dei tratti caratteristici di una pittura.

Proprio perché l'epigramma del bellus homo è esemplare del modo di poetare di Marziale, individuare in esso alcuni codici retorici significa capire il contributo che la retorica stessa dà all'estro creativo di Marziale; significa poter entrare nel laboratorio e apprezzare i procedimenti attraverso i quali egli realizza la sua vivida e spiritosa poesia, almeno per quanto riguarda gli aspetti espressivi legati alla rappresentazione realistica e comica dei personaggi.

Una chiave d'accesso alla valutazione e al riconoscimento dei codici retorici dell'epigramma ci è fornito, in questo senso, da un dato esegetico già noto agli studi di Marziale: l'accostamento tra le divertenti descrizioni di vizi e difetti che leggiamo in Marziale, e la famosa galleria di tipi umani, o Caratteri, descritti da Teofrasto. Una certa affinità di forme e movenze espressive avvicina i due testi: il tono scherzoso, niente affatto moralistico, dei ritratti, la loro natura tipologica, la descrizione particolareggiata, l'idea di raffigurare il personaggio attraverso i suoi comportamenti caratteristici, la costruzione di piccoli sketches da cui si rivela con immediatezza l'indole dell'uomo, l'attenzione a rappresentare scene di vita quotidiana, riprodotte con realismo. E a proposito del bellus homo si è già notata la contiguità di contenuti con gli

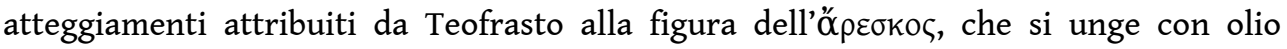
profumato, cura molto il vestiario e frequenta i ginnasi ${ }^{21}$ :

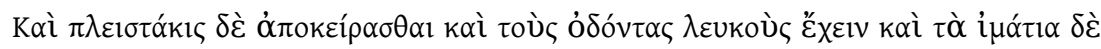

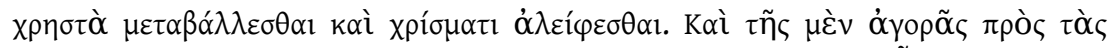

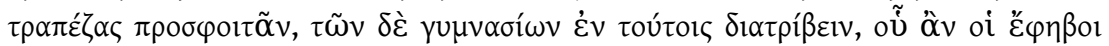

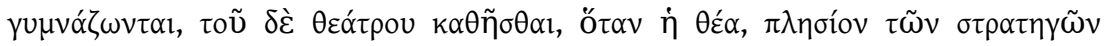
(Theophr., char. 5, 6-7).

«Il piacente si taglia spessissimo barba e capelli, e tiene i denti bianchi, e smette vestiti ancora buoni e si unge con unguento profumato. E frequenta quella parte della piazza dove sono i cambiavalute, e passa ore e ore in quei ginnasi dove fanno istruzione gli efebi, e, quando c'è rivista, siede in teatro vicino agli strateghi » (trad. G. Pasquali).

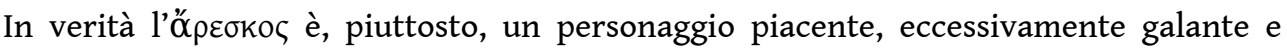
cortese, che si prodiga di continuo in complimenti e smancerie solo per il gusto di piacere all'interlocutore. Si tratta di un tipo umano un po' diverso dal dandy affettato di Marziale. E anche bellus pare una traduzione poco precisa dell'aggettivo greco. Ma queste incongruenze possono risolversi se teniamo presente che il testo trasmesso da Teofrasto è molto corrotto e nel capitolo dedicato alla $\dot{\alpha} \rho \varepsilon ́ \sigma \kappa \varepsilon l \alpha$ i filologi individuano la conflazione di due diversi caratteri, con una cesura dal par. 6 in poi, laddove sembra iniziare la descrizione di un altro tipo umano, diverso dall'uomo piacente, più vicino al bel tipetto di Marziale ${ }^{22}$. 
16 Aver riconosciuto nei Caratteri di Teofrasto un modello di riferimento per la descrizione tipologica di Marziale, ci permette di approfondire la ricerca dei codici retorici ellenistici impiegati dal poeta per i suoi epigrammi. I Caratteri di Teofrasto costituiscono, infatti, un'opera singolare, per la quale ogni etichetta di genere letterario finora impiegata appare restrittiva. Alcuni vi hanno visto una raccolta di appunti per un corso di etica descrittiva ${ }^{23}$, altri uno scritto di poetica che illustra $i$ personaggi della commedia, in particolare quella nuova, con cui condivide l'aspetto tipologico dei protagonisti ${ }^{24}$, altri, infine, vi hanno individuato una chiara finalità retorica: i Caratteri di Teofrasto sarebbero un testo di supporto per le esercitazioni scolastiche, un prontuario di bozzetti che servono da modello all'aspirante oratore per la caratterizzazione dei personaggi ${ }^{25}$.

Non sappiamo se i Caratteri avessero originariamente una finalità filosofica, poetica o retorica; quello che però sappiamo è che, di certo, la fortuna e il riuso cui andò incontro il testo di Teofrasto furono di stampo retorico e che in età ellenistica esisteva una

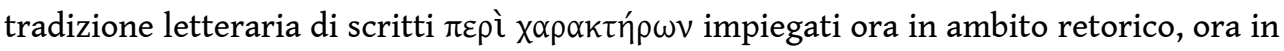
ambito filosofico. La tradizione manoscritta ha trasmesso i Caratteri all'interno di codici di argomento retorico così come i ritratti di personaggi-tipo descritti da Teofrasto hanno costituito il punto di riferimento per l'elaborazione di figure come quella di Pisone nell'omonima orazione di Cicerone o di tanti altri personaggi nelle storie di Sallustio e Tacito: si tratta di segni inequivocabili del reimpiego di questi testi presso le scuole retoriche ${ }^{26}$. Analogamente ci sono tracce di un filone letterario attivo già in età ellenistica, più incline di certo a interessi etico-filosofici, tutto interno alla scuola peripatetica, ma non esclusivo, inaugurato da Teofrasto e proseguito da altri intellettuali come Aristone di Chio, Eraclide Pontico e Satiro di Callati, autori di testi

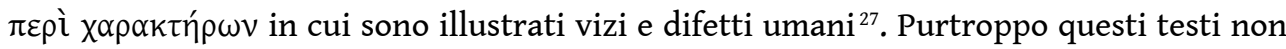
ci sono pervenuti, ma dalle poche testimonianze indirette (la principale delle quali è fornita da Filodemo che nel de vitiis cita stralci dall'opera di Aristone di Chio) ${ }^{28}$ possiamo dedurre che tali scritti avevano una struttura-standard: essa prevedeva la definizione iniziale del vizio, fissato nel tipo umano corrispondente (come ad es. l'avaro, il parassita, il borioso, etc.), cui faceva seguito la descrizione dei comportamenti tipici attraverso una serie di scenette vivaci, tratte dalla vita quotidiana ${ }^{29}$.

Pur non conoscendo con precisione le linee di sviluppo di questa tipologia di scritti, tuttavia siamo informati degli esiti che possiamo rinvenire nelle fonti a partire dal I sec. a.C. e che sono di natura essenzialmente retorica. Nella Rhetorica ad Herennium, il primo manuale in lingua latina a noi noto, ritroviamo il ritratto di un ostentatore di ricchezze come esempio di notatio, la figura retorica che consiste nella descrizione del carattere di un individuo attraverso alcuni segni tipici che, come notae, fanno riconoscere la sua indole. Torneremo su questa figura quando vorremo delineare con precisione le forme espressive e i procedimenti tipici che realizzano la descrizione del carattere. Per ora rileviamo, insieme a Calboli ${ }^{30}$, l'aspetto tipologico del personaggio, l'affinità di alcuni suoi tratti con il carattere teofrasteo dell'ỏ $\lambda \alpha \zeta o v \varepsilon i ́ \alpha$, e il tono di bonaria ironia che distingue il racconto, tipico della commedia menandrea. Cicerone nota come si possa discutere della definizione di un argomento - ad es. cos'è l'avarizia mediante una descrizione del tipo umano corrispondente - chi è l'avaroraffigurandolo nel suo comportamento consueto; è la descrizione della vita e della natura di un uomo, cosa che i Greci chiamano $\chi \alpha \rho \alpha \kappa \tau \eta ́ p \rho^{31}$. Alla seconda metà del I 
sec. a.C. risale con buona approssimazione il manuale di figure retoriche di Rutilio Lupo, il quale traduce e rielabora l'analogo lavoro di Gorgia di Atene ${ }^{32}$. Tra le figure di pensiero spicca il characterismos che consiste nella vivida descrizione dei vizi e delle virtù della persona ${ }^{33}$. L'esempio citato da Rutilio è tratto dall'opera di Licone, un filosofo peripatetico, e descrive in una serie di sketches il comportamento dell'ubriacone durante il corso della giornata.

$19 \mathrm{Al}$ I sec. d.C., in un'età vicina a Marziale, risale la testimonianza di Seneca che in una delle sue epistole a Lucilio ricorda come nell'ambito della riflessione filosofica sui vizi e sulle virtù degli uomini, la definizione dei comportamenti giusti e sbagliati possa essere efficacemente sostituita da una loro descrizione tipologica, che alcuni chiamano characterismos, lo stoico Posidonio invece ethologia. Tale descrizione riproduce i segni e le notae di ciascun vizio e di ciascuna virtù ${ }^{34}$. La tradizione di questi scritti non è solo viva all'epoca di Marziale, ma anche in pieno secondo secolo, se è vero che Aulo Gellio interpreta un episodio degli Annales di Ennio leggendovi il ritratto di un uomo che si comporta affabilmente con un amico di rango superiore. Aulo Gellio nota come il poeta abbia rappresentato la natura dell'uomo in maniera così pittorica da offrire un esempio memorabile al pari degli antichi precetti dei filosofi, e manifesta così il suo apprezzamento per questo modo vivace e brillante di trattare argomenti di etica ${ }^{35}$.

La condivisione di uno stesso vocabolario tecnico, i contenuti assai simili, il campionario di esempi, tratti da filosofi peripatetici, tradiscono un rapporto stretto tra i due ambiti dell'etica e della retorica. Non sappiamo se queste descrizioni tipologiche si siano diffuse prima in ambito filosofico e poi retorico o viceversa. Comunque sia, per la nostra indagine è sufficiente evidenziare che in età ellenistica, almeno per quanto ci risulta dalla lettura delle fonti retoriche a nostra disposizione (risalenti al I sec. a.C.),

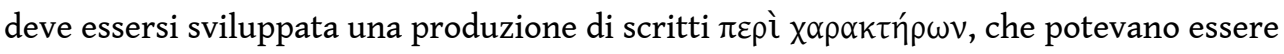
impiegati ora con finalità di parenesi morale, ora, invece, con finalità di esercizi retorici, come repertorio di tipi umani utile alla caratterizzazione dei personaggi nell'ambito delle orazioni ${ }^{36}$. Utile deve essere stata anche l'esperienza della commedia nuova, che forse si serviva di queste considerazioni relative alla descrizione del carattere e al contempo le alimentava ${ }^{37}$. Il ragazzo che studiava retorica, trovava in simili raccolte tipologiche tutte le informazioni necessarie per caratterizzare i suoi personaggi e imparava a scrivere discorsi adatti ai diversi personaggi.

21 Il naufragio pressoché completo delle fonti retoriche di età ellenistica non permette di avere testimonianze dirette di questa tipologia di esercizi. Sappiamo però da Quintiliano che nelle scuole di età imperiale, quindi anche nella scuola di Marziale, era praticato lo studio dei caratteri: i ragazzi imparavano a tracciare le linee dei diversi tipi umani (avari, rozzi, superstiziosi, etc.) e sulla base della loro imitazione elaboravano poi i discorsi ${ }^{38}$. Forse la descrizione del carattere costituì un vero e proprio esercizio di scrittura (progymnasma), se è vero che in Svetonio e soprattutto Quintiliano troviamo l' ethologia accanto a esercizi come l'aneddoto (chria) e la massima (sententia) ${ }^{39}$. Ma le due fonti latine si limitano a citare l'esercizio senza darne un'accurata illustrazione; solo Quintiliano ci dice che esso verteva sulla lettura di modelli e, a differenza della massima che aveva valore universale, si riferiva a persone ${ }^{40}$. Tuttavia, sulla lettura delle due testimonianze gravano problemi di trasmissione testuale e non si è affatto sicuri che la lezione genuina sia ethologia, un termine che richiamerebbe la descrizione dei vizi e delle virtù di cui parla Seneca nel passo della epistola già citato (epist. 95, 65) ${ }^{41}$. 
22 Non è, tuttavia, necessario presupporre l'esistenza dell'ethologia per essere certi che nelle scuole retoriche si insegnasse la descrizione del carattere e che su questa i giovani affinassero le proprie capacità di scrittura. Un altro esercizio, ben più diffuso, di cui abbiamo grande disponibilità di informazioni sia a livello di fonti dottrinarie, sia a livello di esercizi svolti e/o fittizi, aveva nella raffigurazione morale della persona uno degli elementi costitutivi: è l'etopea ${ }^{42}$. Essa consisteva nella simulazione di un discorso diretto, messo in bocca ad un uomo in conformità al suo carattere e alle circostanze. Lo studente componeva l'orazione immaginando le parole che un dato personaggio, preso solitamente dalla mitologia e dalla storia, avesse pronunciato in una determinata occasione. In vero, l'esercizio non prevedeva una descrizione del carattere in forma espositiva, come avveniva nell'opera di Teofrasto; la rappresentazione dell'ethos doveva, invece, trasparire dalle parole che la persona pronunciava. Se Menandro aveva sentenziato che il carattere di una persona si riconosce dalle parole ${ }^{43}$, più tardi, con un'efficace metafora, Dionigi avrebbe affermato che le parole sono vivide immagini del carattere $^{44}$. Nell'etopea, quindi, lo studente, senza l'ausilio di note descrittive doveva ripresentare lo stato d'animo del personaggio facendolo affiorare spontaneamente dal discorso; per questo doveva prima costruire l'ethos della persona e poi mettere sulla sua bocca parole adeguate. Gli studenti usavano a questo scopo le descrizioni tipologiche composte dal maestro o ne stendevano di personali prima di scrivere i loro discorsi.

Del resto, una tradizione retorica ben consolidata tra il isec.a.C. e il Isec.d.C. attribuisce all'etopea, intesa questa volta come figura retorica, il valore di descrizione del carattere, raffigurazione della vita e della natura dell'uomo, da potersi realizzare ora attraverso il discorso (in dictis), ora attraverso i fatti (in factis). Esiste cioè un preciso indirizzo, che ha in Quintiliano il suo rappresentante più significativo e in Cicerone il primo testimone ${ }^{45}$, che assembla in un'unica figura la rappresentazione mimetica del comportamento dell'individuo, riunendo in un'unica fattispecie i due diversi procedimenti della simulazione del discorso e della descrizione analitica del comportamento ${ }^{46}$. L'apprezzata finalità comica, le citazioni tratte dalla commedia dei caratteri di Terenzio, insieme ad alcune considerazioni sulle figure della descrizione di carattere, che Quintiliano riserva in altro luogo ${ }^{47}$, assicurano, a mio avviso, la corrispondenza tra questa figura retorica e la raffigurazione tipologica dei caratteri che si legge in Teofrasto, Rutilio Lupo (characterismos) e la Rhetorica ad Herennium (notatio).

È venuto il momento di tirare le somme di questo lungo excursus nella tradizione retorica: abbiamo trovato codici retorici ben definiti cui poter ricondurre i Caratteri di Teofrasto e, per loro tramite, l'epigramma di Marziale del bellus homo, da cui siamo partiti. Abbiamo cioè appurato che nella scuola, frequentata dal poeta, erano studiate fattispecie retoriche, vuoi in forma di esercizio scolastico, vuoi come figure di pensiero, che servivano a descrivere le qualità e i difetti morali di un individuo.

Ora dobbiamo compiere un passo in avanti: dimostrare se Marziale ha tenuto presente questi codici retorici nella raffigurazione del bellus homo, capire quali procedimenti espressivi ha usato e per quali finalità. Possiamo verificare l'influenza del modello retorico solo apprezzando la condivisione di tecniche narrative, procedimenti espressivi, struttura ed elementi formali tra l'epigramma e queste fattispecie. Risulta dunque opportuna e funzionale alla nostra indagine una rilettura della tecnografia per definire le caratteristiche di questo particolare 'codice retorico'. Ci serviremo essenzialmente di due testimonianze, quella della Rhetorica ad Herennium, che illustra la figura di pensiero della notatio, e quella di Rutilio Lupo, che parla di characterismos. I due 
testi riguardano certamente lo stesso meccanismo espressivo, sebbene vi siano dubbi sulla perfetta equivalenza tra i due termini tecnici (non siamo sicuri, cioè, che notatio

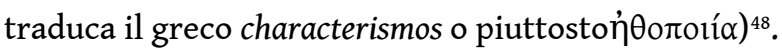

Che cosa ci dicono dunque le due fonti retoriche?

Il procedimento espressivo consiste nella raffigurazione del carattere e della natura di un uomo realizzata mediante la descrizione di comportamenti tipici che, come segni, rivelano i vizi e le virtù dell'individuo. Possiamo trovare alcuni elementi costanti nella definizione dei retori:

- La descrizione è tipologica, ovvero organizzata intorno a tipi umani esemplari (come l'ostentatore di ricchezze o l'ubriacone $)^{49}$.

- Proprio perché tipologica, la descrizione si serve di luoghi comuni; bisogna descrivere ciò che è consentaneo alla natura dell'individuo, attingendo da un vasto repertorio di atteggiamenti consueti e ricorrenti osservati nella realtà e codificati da una vasta letteratura (commedia, filosofia) ${ }^{50}$.

- A livello formale la descrizione è organizzata in una serie di sketches, brevi scenette in cui si rivela il comportamento tipico dell'individuo. La descrizione è molto dettagliata e si appunta con dovizia di particolari sulle azioni, sulle parole e sull'aspetto persino fisico del personaggio.

- La descrizione ha una struttura standard, rigida e ripetitiva. I retori non la illustrano, ma possiamo ricavare le informazioni dagli esempi citati (il lungo brano della Rhetorica ad Herennium, ma anche le seppur esigue testimonianze tratte dai testi di età ellenistica di Aristone di Chio o Satiro di Callati). Solitamente la descrizione è introdotta dalla formula:

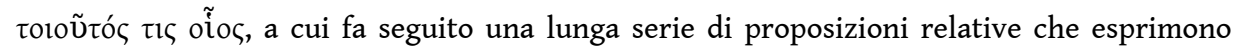
l'azione svolta dal personaggio, mentre costrutti al participio indicano le circostanze di svolgimento dell'azione ${ }^{51}$. La descrizione è dettagliata e ritrae il comportamento dell'uomo nell'arco dell'intera giornata, nelle più disparate situazioni. La struttura rigida e ripetitiva si addice ad un esercizio scolastico.

- La descrizione è vivida. La cura per il dettaglio, l'accumulo dei particolari, è funzionale ad una rappresentazione icastica che ricostruisca l'immagine dell'individuo ponendolo dinanzi agli occhi del lettore/ascoltatore ${ }^{52}$. La Rhetorica ad Herennium rileva nella evidenza visiva l'effetto della descrizione tipologica, usando l'espressione ante oculos ponere, con cui si

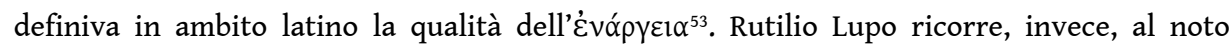
paragone con la pittura: come un pittore si serve dei colori per dipingere le figure, riuscendo persino ad esprimere nei tratti del viso le qualità morali del personaggio, così l'oratore tratteggia le figure di coloro di cui parla ${ }^{54}$. La descrizione del carattere è evidente ed essa stessa è strumento di evidenza ${ }^{55}$.

- A questa vivezza espressiva non è di ostacolo, anzi è funzionale la descrizione di motivi topici. Infatti, l'impressione di evidenza visiva scaturisce dalla rielaborazione di immagini depositate nella memoria di chi legge o ascolta. Il riferimento a luoghi comuni non inaridisce l'immediatezza della descrizione, piuttosto ne favorisce l'attivazione, perché grazie al rimando a situazioni e sensazioni già vissute, il lettore (o l'ascoltatore) può immaginare di trovarsi nelle stesse circostanze e reagire con la medesima partecipazione emotiva ${ }^{56}$.

- La descrizione è piacevole: la Rhetorica ad Herennium individua nella delectatio l'altro effetto della notatio ${ }^{57}$; analogamente Quintiliano ricorda che il ricorso all'etopea avviene soprattutto con finalità comiche (in eludendo) ${ }^{58}$. 
- La descrizione è realistica. L'impressione complessiva che si ricava dalla lettura di simili brani è quella di essere dinanzi alla realtà vissuta. Lo storico e retore Dionigi di Alicarnasso fa dell'etopea una qualità dello stile e individua in Lisia l'oratore che più di ogni altro ha saputo ritrarre il comportamento degli uomini, costruendo orazioni adatte alla loro personalità. È la rappresentazione del carattere, così verosimile, a rendere i suoi discorsi tanto credibili e persuasivi ${ }^{59}$.

28 Abbiamo in questo modo individuato le caratteristiche formali e le finalità espressive che definiscono la descrizione del carattere. Dobbiamo ora verificare il loro riuso da parte di Marziale nell'epigramma del bellus homo.

Se di certo l'elemento più evidente risulta essere l'estesa descrizione tipologica che occupa dieci dei quattordici versi dell'epigramma e che ritrae il comportamento del dandy attraverso tante scenette in sé autonome, secondo la tipica tecnica teofrastea, come è stato già rilevato dagli studiosi, a mio avviso l'adesione al canone retorico della notatio o characterismos si rivela soprattutto nella struttura. L'epigramma ha una richiesta di definizione iniziale, seguita dalla descrizione tipologica. Questa descrizione ripropone in maniera identica la formula fissa di apertura degli esercizi retorici grazie al pronome relativo qui, secondo l'esempio: il personaggio è tale che ... Lo schema si ripete costante nel corso della descrizione, mediante l'anafora martellante del relativo. La sintassi rimane identica e le scenette sono coordinate per asindeto, presentando la stessa articolazione del carattere teofrasteo. A conclusione della descrizione viene data a sorpresa la definizione, ma il passaggio è introdotto da una formula (quid narras?) che appare ancor più interessante dopo la lettura della Rhetorica ad Herennium ${ }^{60}$. In modo analogo si conclude, infatti, il brano citato dal manuale; e il riferimento al narrare, qui inteso nel valore di 'descrivere', è molto puntuale secondo un'accezione quasi 'retorica' e la formula 'quid narras?' mostra i debiti contratti con questi esercizi ${ }^{61}$.

L'adesione a questi modelli retorici da parte di Marziale può apprezzarsi mediante il confronto con le altre rielaborazioni sul motivo del bellus homo o con le altre descrizioni tipologiche presenti nella letteratura latina. Possiamo prendere a riferimento per esempio Catullo ${ }^{62}$, Orazio ${ }^{63}$ o, altrove, lo stesso Marziale ${ }^{64}$. Ebbene, persino nei ritratti morali più accurati, persino in quelli per i quali i commentatori antichi non hanno esitato ad individuare la figura della descrizione del carattere (o characterismos) ${ }^{65}$, non si trova una così stretta corrispondenza di formule e schemi espressivi. Segno che il riferimento a precisi 'codici retorici' perseguito qui da Marziale è cercato, voluto.

31 Marziale avrebbe potuto in effetti parlare del bellus homo come fa in un breve epigramma del primo libro, in cui la battuta finale ridimensiona l'altezzosità dell'uomo vanitoso definendolo pusillus homo ${ }^{66}$. 0 avrebbe potuto elencare tutti i suoi comportamenti, come fa nell'epigramma 2,7, dove alcuni elementi (la struttura sintattica fissa, l'anafora in poliptoto di bellus, l'accumulo dei tratti morali in brevissime scenette) ricordano tonalità e movenze espressive dell'epigramma 3,6367. O ancora avrebbe potuto creare un gioco di parole sull'epiteto bellus e il nome del povero malcapitato confezionando una feroce pointe finale ${ }^{68}$. Invece, egli ripropone lo schema espositivo e i procedimenti espressivi tipici del characterismos o della notatio.

Tornerò su questo aspetto in sede di conclusione per suggerire una semplice suggestione che scaturisce da tali considerazioni. Per ora non vorrei abbandonare l'esegesi retorica dell'epigramma di Marziale senza prima notare che proprio la decodificazione dei modelli retorici della notatio e del characterismos consente di rileggere la poesia, reinterpretando molti elementi già noti alla tradizione esegetica. La 
notevole espansione che interessa il momento descrittivo della poesia di Marziale non è solo funzionale a creare l'attesa per la battuta finale, ma è, almeno in questo caso, strumento per realizzare effetti di evidenza visiva. Marziale dipinge il ritratto del bellus homo grazie alla dovizia dei particolari. L'accumulo dei dettagli, che si susseguono nel ritmo serrato della coordinazione per asindeto, consente al lettore di ricreare l'immagine di questo piccolo uomo e l'anafora del pronome enfatizza questo aspetto ${ }^{69}$. Anche un certo ordine visivo, con cui sono proposte al lettore le diverse scenette (secondo una tecnica di progressiva dissolvenza per cui si parte dallo zoom iniziale sulla figura del bellus homo, un primo piano sui capelli, sulla bocca e sulle braccia, per poi passare a una ripresa più ampia), è funzionale per seguire il percorso visivo di osservazione del poeta e ricostruire l'immagine ${ }^{70}$. E persino la tipicità di alcuni atteggiamenti, come il pettinarsi con cura i capelli, il cospargersi di profumo, il depilarsi le braccia, che ritroviamo anche in altre variazioni sul tema del bellus homo elaborate dai poeti latini e dello stesso Marziale ${ }^{71}$, favorisce l'attivazione del processo di immaginazione: richiamando alla memoria analoghe situazioni ed esperienze di vita, ci consente di vedere all'opera questo personaggio mentre fa il gagà nei salotti dell'alta società $^{72}$.

\section{Conclusioni}

La ricerca di codici retorici nell'epigramma di Marziale ha dato esiti positivi: il ritratto del bellus homo è condotto prendendo a riferimento l'esercizio retorico della descrizione di carattere. Nei versi ritroviamo i procedimenti espressivi, le tecniche di esposizione, gli elementi formali tipici di questa fattispecie retorica. La struttura della descrizione e la formula finale del quid narras? manifestano la ricercata corrispondenza con questa forma narrativa che Marziale ha sicuramente praticato nel suo tirocinio scolastico. D'altronde, anche altrove il poeta mostra di conoscere con precisione la dottrina retorica, persino nell'attualità della terminologia tecnica ${ }^{73}$. Resta da chiedersi il perché di una simile scelta e quali novità esegetiche apporta questo riconosciuto influsso retorico sull'interpretazione dell'epigramma di Marziale e in generale sul suo modo di fare poesia.

34 A mio giudizio, l'individuazione di un modello retorico ripreso e rielaborato aggiunge alla pointe finale ulteriore forza espressiva. Il lettore colto, educato alla scuola dei retori, riconosce subito che l'intelaiatura dell'intero componimento si basa sugli schemi compositivi delle descrizioni di carattere e che, alla fine, questa struttura è scardinata dall'aprosdoketon conclusivo. Ai versi viene ad aggiungersi un valore in qualche modo parodico e l'effetto comico scaturisce non solo dalla insulsa definizione del personaggio (res pertricosa), ma dal ribaltamento del modello retorico ${ }^{74}$. Sembra quasi - ma è solo una suggestione - che la comicità elegante e garbata di Marziale si appunti anche contro una certa figura di retore, molto vicina al dandy qui descritto: un uomo colto e affettato, che fa esibizione della sua eleganza. Nell'epigramma 2, 7 prima ricordato, il protagonista, Attico, è uno di questi: declama in modo elegante, tiene arringhe in modo elegante, racconta storie, compone poesie e scrive mimi in modo elegante, è un grammatico elegante. Fa tutto in modo elegante: che cos'è? È un ... casinista! Nella categoria dei belli homines, il retore deve avere un posto privilegiato. Roma conosce molti di questi personaggi: sono intellettuali vanitosi, declamatori da strapazzo che fanno sfoggio di una vasta erudizione su argomenti frivoli, salottieri, amanti del lusso e 
dei pettegolezzi ${ }^{75}$. Il ritratto di uno di questi ci è offerto da Luciano nel Praeceptor rhetorum: è un uomo tutta sapienza e bellezza, che ha l'andatura dinoccolata, la voce flautata, i capelli curati, la pelle profumata ${ }^{76}$.

Se, dunque, l'indagine retorica permette di trovare nuove chiavi di lettura dell'epigramma, nondimeno essa è utile per entrare nel laboratorio poetico di Marziale e capire la sua scrittura. Dall'analisi retorica del testo, infatti, abbiamo potuto notare come quel realismo e quella freschezza di immagini, per cui l'epigramma di Marziale è giustamente celebre, scaturiscono da un'attenzione particolare ai meccanismi espressivi della visualizzazione e da una sapiente caratterizzazione del personaggio. Più volte lodato per le sue qualità di immediatezza espressiva, apprezzato ora per i vividi bozzetti, ora per la vivace rappresentazione della società romana, l'epigramma di Marziale deve le sue qualità ad un sapiente uso dei procedimenti dell'evidenza testuale. In un'epoca che si interroga sul rapporto tra parola e immagine, sulla capacità della parola di eguagliare la pittura e la sua visività, sulla possibilità di costruire personaggi credibili e di rappresentare nella poesia uomini e donne reali con le loro emozioni e i loro caratteri ${ }^{77}$, l'operazione letteraria messa in atto da Marziale non pare casuale: il poeta sembra proporre la sua soluzione niente affatto ingenua, niente affatto spontanea, in un epigramma vivido che assume le forme e il tono di un nitido ritratto.

Sarebbe opportuno estendere l'analisi all'intera raccolta del poeta per ricercare, direi quasi 'vedere', se i codici retorici dell'evidenza espressiva e della descrizione del carattere siano elemento fondamentale dello stile del poeta e possano spiegare il suo noto realismo. Dallo studio del ritratto del bellus homo possiamo comunque trarre la conclusione che al realismo di Marziale non sia affatto estranea la retorica, ma che piuttosto sia la retorica stessa a nutrire la sua musa fornendo gli strumenti per restituire nei versi l'impressione della vita e del quotidiano ${ }^{78}$. I meccanismi dell'evidenza retorica, che pone i fatti davanti agli occhi dei lettori / ascoltatori ${ }^{79}$, servono a costruire questo «tableau de la vie», che «nous mettent sous les yeux la vie de tous les jours» ${ }^{80}$. Solo che il risultato è spontaneo, l'arte dissimulata e tutto appare così naturale ${ }^{81}$.

Perciò non trovo migliore descrizione della poesia di Marziale se non adattando il giudizio che Dionigi di Alicaranno aveva formulato sulle orazioni di Lisia, così popolate di personaggi reali e piene di vita vera: se vuoi riprodurre la verità e vuoi essere imitatore della natura, non sbaglieresti a servirti della tecnica compositiva di Marziale; infatti, non troveresti nessun'altra poesia più vera di questa! ${ }^{82}$

\section{BIBLIOGRAFIA}

\section{Edizioni}

Cornifici, Rhetorica ad C. Herennium, introd., testo critico, commento a cura di G. CALBoLI, Edizioni e saggi universitari di filologia classica 11, Bologna, R. Pàtron, 1969. 
Martial, Épigrammes. I, Livres I-VII, texte établi et trad. par H. J. IZAAC, CUF, Paris, Les Belles Lettres, 1969.

Marziale, Epigrammi, I, a cura di S. BETA, Classici greci e latini 85, Milano, A. Mondadori, 1995.

M. Valerii Martialis Epigrammaton liber tertius, introd., ed. critica, trad. e commento a cura di A. FUSI, Spudasmata 108, Hildsheim, G. Olms, 2006.

P. Rutilii Lupi Schemata dianoeas et lexeos, saggio introd., testo e trad. a cura di G. BARABINO, Pubblicazioni dell'Istituto di filologia classica e medievale dell'Università di Genova 40, Genova, Istituto di filologia classica e medioevale, 1967.

Suétone, Grammairiens et rhéteurs, texte établi et trad. par M.-C. VACHER, CUF, Paris, Les Belles Lettres, 1993.

Aelius Théon, Progymnasmata, texte établi et trad. par M. PATILLon, CUF, Paris, Les Belles Lettres, 1997.

Teofrasto, I Caratteri, trad. da G. Coppola, Milano, Mondadori, 1945.

Teofrasto, I Caratteri, testo, introd., trad. e commento di G. PASQUALI, Firenze, Sansoni, 1956.

The Characters of Theophrastus, ed. with introd., commentary and index by R. G. USSHER, London, McMillan, 1960.

The Characters of Theophrastus, newly ed. and transl. by J. M. EDMONDs; Herodes, Cercidas, and the Greek Choliambic poets (except Callimachus and Babrius), ed. and transl. by A. D. KNOx, The Loeb Classical Library, Cambridge, W. Heinemann, 1961.

Teofrasto, Caratteri, introd., trad. e note di L. TORRACA, Milano, Garzanti, 1994.

Theophrastus, Characters; Herodas, Mimes; Sophron and other Mime Fragments, ed. and transl. by J. RUSTEN, I. C. CUNNINGHAM, The Loeb Classical Library. Greek Authors 225, Cambridge, Harvard University Press, 2002.

Theophrastus, Characters, ed. with introd., transl. and commentary by J. DIGGLE, Cambridge, Cambridge University Press, 2004.

\section{Studi}

AMATO E., SCHAMP J. (ed.) 2005, Ethopoiia. La représentation de caractères entre fiction scolaire et réalité vivante à l'époque imperiale et tardive, Cardo 3, Salerno, Helios.

BARTWICK K. 1959, Martial und die zeitgenössische Rhetorik, Berlin, Akademie-Verlag.

BERARDI F. 2010, «La descrizione dello spazio: procedimenti espressivi e tecniche di composizione secondo i retori greci», in C. Jarruesco (ed.), Topos-Chôra: l'espai a Grècia. I, Perspectives interdisciplinàries. Homenatge a Jean-Pierre Vernant $i$ Pierre Vidal-Naquet, Tarragona, Institut català d'arqueologia clàssica, p. 37-48.

- 2012, La dottrina dell'evidenza nella tradizione retorica greca e latina, Perugia, Pliniana.

- 2013, «L'ethologia e l'aetiologia nei progymnasmata in lingua latina», Rétor 3, p. 122-148.

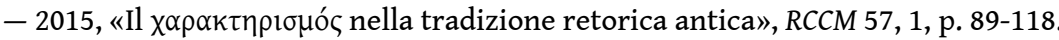

BRUSS K. S. 2013, «Persuasive Ethopoeia in Dionysius's Lysias», Rhetorica 31, 1, p. 34-57.

BORGo A. 2003, Retorica e poetica nei proemi di Marziale, Studi latini 51, Napoli, Loffredo. 
CALBOLI G. 1998, «From Aristotelian Lexis to elocutio», Rhetorica 16, 1, p. 47-80.

CALBOLI MONTEFUSCO L. 1996, "Quintilian and the Function of the Oratorical exercitatio», Latomus 55, p. 615-625.

CELENTANO M. S. 1999, «Parody between Rhetoric and Literature», in L. CALBoli MONTEFUsCo (ed.), Papers on Rhetoric, II, Bologna, CLUEB, p. 99-105.

- 2003, s.v. «Paradoxon», in G. Ueding (Hrsg.), Historisches Wörterbuch der Rhetorik, VI, Tübingen, M. Niemeyer, coll. 524-526.

- 2010, «Quintiliano e la duplice exercitatio nell'Institutio oratoria», in L. BRISSON, P. CHIRON (ed.), Rhetorica philosophans. Mélanges offerts à Michel Patillon, Textes et traditions 20, Paris, J. Vrin.

CITRONI M. 1989, «Marziale e la letteratura per i Saturnali (poetica dell'intrattenimento e cronologia della pubblicazione dei libri)», ICS 14, p. 201-226.

CIZEK A. 1994, Imitatio et Tractatio. Die literarisch-rhetorischen Grundlagen der Nachahmung in Antike und Mittelalter, Rhetorik-Forschungen 7, Tübingen, M. Niemeyer.

CRIBIORE R. 2001, The Gymnastics of the Mind. Greek Education in Hellenistic and Roman Egypt, Princeton, Princeton University Press.

DE TEMMERMAN K. 2004, «Caractérisation et discours direct : le cas de Plangon», in B. POUderon, J. PEIGNEY (ed.), Discours et débats dans l'ancien roman. Actes du colloque de Tours 21-23 octobre 2004, CMO 36, Lyon, Maison de l'Orient et de la Méditerranée, p. 63-75.

- 2010, «Ancient Rhetoric as a Hermeneutical Tool for the Analysis of Characterization in Narrative Literature», Rhetorica 28, 1, p. 23-51.

DUBEL S. 1997, «Ekphrasis et enargeia : la description antique comme parcours», in C. LÉVY, L. PERNOT (ed.), Dire l'évidence. Philosophie et rhétorique antiques, Cahiers de philosophie ancienne et du langage de l'Université de Paris XII-Val-de-Marne 2, Paris, L'Harmattan, p. 249-264.

ESTELLÉS GONZÁLEZ J. M. 2009, «El poeta latino Marcial u sus cosas: un "bellus homo" o "el hábito non hace al monje"», in C. BENOIT et all. (ed.), Homenaje a Dolores Jiménez Plaza. Escrituras del amor y del erotismo, Valencia, Facultat de Filologia, Traducció i Comunicació, Universidad de Valencia, p. $113-120$.

FORTENBAUGH W. W. 1984, Quellen zur Ethik Theophrasts, Studien zur Antiken Philosophie 12, Amsterdam, B. R. Grüner.

GAISER K. 1967, «Menander und der Peripatos», A\&A 13, p. 8-40.

GARVER E. 2000, «'H HOऽ and Argument», in L. CALBOLI MONTEFUSCO (ed.), Papers on Rhetoric, III, Bologna, CLUEB, p. 113-126.

GILL C. 1983, «The Question of Character-Development. Plutarch and Tacitus», CQ 33, p. 469-487.

GRANATELLI R. 1995, «M. Fabio Quintiliano Institutio oratoria II 1-10: struttura e problemi interpretativi», Rhetorica 13, 2, p. 137-160.

HEATH M. 2003, «Theon and the History of the Progymnasmata», GRBS 43, p. 129-160.

HUNTER R. 1985, The New Comedy of Greece and Rome, Cambridge, Cambridge University Press.

KENNEDY G. A. 1999, Classical Rhetoric and its Christian and Secular Tradition from Ancient to Modern Times, 2nd ed., Chapel Hill, University of North Carolina Press. 
KRAUS M. 2005, s.v. «Progymnasmata, Gymnasmata», in G. UEDING (Hrsg.), Historisches Wörterbuch der Rhetorik, VII, Tübingen, M. Niemeyer, coll. 159-167.

- 2007, «Rehearising the Other Sex: Impersonation of Women in Ancient Classroom Ethopoiia», in J. SALAMANCA, J. A. FERNÁNDEZ DELGADO, F. PORDOMINGO, A. STRAMAGLIA (ed.), Escuela y literatura en Grecia antigua. actas del simposio internacional, Universidad de Salamanca, 17-19 noviembre de 2004, Cassino, Ed. dell'Università degli studi di Cassino, p. 455-468.

KRUUSE J. 1941, «L'originalité artistique de Martial», C\&M 4, p. 248-300.

LAUSBERG H. 1960, Handbuch der literarischen Rhetorik. Eine Grundlegung der Literaturwissenschaft, München, Max Hueber, 2 vol.

LESSING G. E. 2009, Osservazioni sparse sull'epigramma e alcuni dei più distinti epigrammatisti, Nuovo medioevo 78, Napoli, Liguori Editore [Zerstreute Anmerkungen über das Epigramm und einige der vornehmsten Epigrammatisten, Wien, 1771].

MANIERI A. 1998, L'immagine poetica nella teoria degli antichi. Fantasia ed enargeia, Filologia e critica 82, Pisa, Istituti editoriali e poligrafici internazionali.

MARCHESI C. 1940, Valerio Marziale, 2a ed., Milano, Bietti.

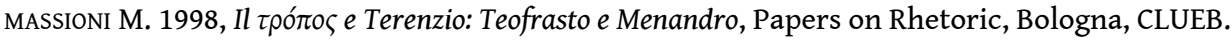

MATELLI E. 1989, «Libro e testo nella tradizione dei Caratteri di Teofrasto», Scrittura e civiltà 13, p. 329-386.

MORETTI G. 2010, «Xenia e Apophoreta di Marziale fra ekphrasis retorica e tradizione iconografica della 'natura morta'», in L. BELLONI et all. (ed.), Le immagini nel testo, il testo nelle Immagini. Rapporti fra parola e visualità nella tradizione greco-latina, Labirinti 128, Trento, Università degli studi di Trento, Dipartimento di studi letterari, linguistici e filologici, p. 327-372.

MORGAN T. 1998, Literate Education in the Hellenistic and Roman Worlds, Cambridge Classical Studies, Cambridge, Cambridge University Press.

PICONE G., ROMANO E., GASTI F. 2003, Lezioni Romane. Letteratura, testi, civiltà, III, Torino, Loescher. PIROVANO L. 2008, «L'insegnamento dei “progymnasmata” nell'opera di Emporio retore», in F. GASTI, E. ROMANo (ed.), Retorica ed educazione delle élites nell'antica Roma. Atti della VI Giornata ghisleriana di filologia classica, Pavia, 4-5 aprile 2006, Studia ghisleriana, Pavia, Collegio Ghislieri, p. 195-236.

RABAU S. 1995, «Narration et description : l'exigence de détails», Lalies 15, p. 273-290.

REICHEL G. 1909, Quaestiones progymnasmaticae, Diss.: Leipzig.

ROBINSON R. P. 1920, «Ethologia or Aetiologia in Suetonius De grammaticis c. 4 and Quintilian 1, 9», CPh 15, 4, p. 370-379.

SCHINDEL U. 1999, Ein unidentifiziertes „Rhetorik-Exzerpt" der lateinische Theon, Göttingen, Vandenhoeck \& Ruprecht.

SMITH K. F. 1920, Martial. The Epigrammatist and other Essays, Baltimore, John Hopkins Press.

THEIN K. 2008, «La force du vraisemblable. Philostrate, Callistrate et l'image à l'epreuve de l'ekphrasis», Metis N.S. 6, p. 315-344.

VALDÉS GARCíA M. A. 2008, «La etopeya en Basilio de Cesarea», Nova Tellus 26, p. 179-200. 
VAN MAL-MAEDER D. 2006, «Lucius descripteur : quelques remarques sur le livre 11 des Métamorphoses d'Apulée», in W. H. KEULEN, R. R. NAUTA, S. PANAYOTAKIS (ed.), Lectiones scrupulosae. Essays on the Text and Interpretation of Apuleius' "Metamorphoses" in honour of Maaike Zimmerman, AncNarr. Suppl. 6, Groningen, Barkhuis Publishing - Groningen University Library, p. 252-265. WEBB R. 2001, «The Progymnasmata as Practice», in Y. L. TooToo (ed.), Education in Greek and Roman Antiquity, Leiden, Brill, p. 289-316.

WEHRLI F. 1968 (Hrsg.), Herakleides Pontikos, Die Schule des Aristoteles 7, Basel, Schwabe \& Co.

ZANGARA A. 2007, Voir l'histoire. théoriesanciennesdurécithistorique, II' siècle avant J.-C. - II ${ }^{e}$ siècle après J.-C., Contextes, Paris, Éd. de l'École des hautes études en sciences sociales - Vrin.

\section{NOTE}

1. Per queste ed altre considerazioni sullo stile e, in generale, sulla poetica di Marziale, vd. CITRONI 1989; BETA 1995; oltre ai fondamentali SMITH 1920; MARCHESI 1940. Una battuta dell'epigramma 8, 3 riassume bene il tono sapido e i contenuti realistici della poesia di Marziale (vv. 19-20): at tu Romano lepidos sale tinge libellos: / adgnoscat mores vita legatque suos, «ma tu condisci con lo spirito romano i tuoi piacevoli libretti: la vita riconosca e legga i suoi costumi».

2. Mart. 10, 4, 8: Hoc lege, quod possit dicere vita "meum est", «leggi ciò di cui la vita possa dire: "è cosa mia"».

3. MARCHESI 1940, p. 63.

4. Potremmo ricordare, a tal proposito, la nota frase di Schopenhauer: «Je weniger rhetorisch die Poesie ist, desto besser», ovvero «La poesia è tanto migliore quanto meno è retorica» (Die Genesis des Systems, im Auftrage und unter Mitwirkung von P. DEUSSEN, Arthur Schopenhauers sämtliche Werke 11, München, R. Piper, 1916, pp. 108-109).

5. Sul rapporto tra letteratura e retorica, vd. KENNEDY 1999, p. 3; il prevalere dell'insegnamento retorico nell'apprendistato della scrittura è la 'retoricizzazione' della letteratura: vd. CIZEK 1994, p. 237.

6. Sul tirocinio alla scrittura presso gli antichi e, più in generale, sull'exercitatio retorica, vd. CALBOLI MONTEFUSCO 1996; CELENTANO 2010.

7. Riguardo agli esercizi preliminari di scrittura (progymnasmata), vd. WEBB 2001; KRAUS 2005.

8. L'istruzione progimnasmatica è propedeutica alla formazione dell'oratore (Theon 2, 1-3), ma è funzionale anche alla preparazione di tutti gli uomini di lettere: Theon 15 , 24-29. Sulla datazione di Teone al i sec. d.C., vd. PATILLON 1997, pp. IX-XIV; non è d'accordo HEATH 2003, pp. 141-158.

9. Un panorama delle fonti latine relative ai progymnasmata (praeexercitamina nella traduzione latina) è offerto da PIROVANO 2008, pp. 195-196. Escludendo la traduzione del manuale di Ps.Ermogene, curata da Prisciano (vi sec.d.c.), i principali testi di riferimento sono Quint. inst. 1, 9; 2, 4; Svet. gramm.4, 6-7; 25, 8. A questi possono aggiungersi due anonimi capitoletti (de laude, de comparatione) costituenti il cosiddetto ‘Teone latino' (vd. ScHINDEL 1999), alcuni capitoli delle Origines di Isidoro (1, 24-25; 2, 
10-14); un capitolo de chria presente nel codice Vat. Lat. 5216 (= GLK 6, 246-247); e, infine, quel che resta del manuale di Emporio (RLM 561-569 Halm).

10. Tracce di dottrina progimnasmatica si rinvengono in Cic. inv. 1, 27; de orat. 1, 54; 154-155; Rhet. Her. 1, 12; 2, 47; 3, 15; 4, 57 (per cui vd. REICHEL 1909, pp. 97-104; LAUSBERG 1960, p. 842. Sull'origine ellenistica del curriculum di studi, testimoniato da reperti papiracei contenenti esercizi svolti, vd. MORGAN 1998; CRIBIORE 2001, pp. 225-230.

11. Cicerone pratica alcuni esercizi, come le theseis, per acquisire l'abilità di scrittura (vd. epist. Att. 9, 4, 1; de orat. 3, 107; Tusc. 2, 9); Quint. inst. 10, 5, 1 ss. prescrive l'uso dei progymnasmata dopo gli studi scolastici per conservare la prontezza nell'elaborazione del discorso e perfezionare la propria capacità di improvvisazione.

12. Riguardo all'influsso esercitato dai progymnasmata su alcuni testi letterari, vd. le recenti indagini di VALDÉS GARCíA 2008 (Basilio di Cesarea), THEIN 2008 (Filostrato), DE TEMMERMAN 2004 (romanzo greco), VAN MAL-MAEDER 2006 (Apuleio).

13. Vd. FUSI 2006, p. 405.

14. L'aggettivo, mai attestato nella letteratura prima di Marziale, è costruito partendo da tricosus, usato ad esempio da Lucilio $(4,17)$ e indicante qualcosa di complicato, pieno di tricae ('difficoltà, fastidi').

15. Lessing (2009) parla di un andamento binario dell'epigramma, dove la prima parte descrive un fatto o un carattere, mentre la seconda ne ricava, attraverso un ragionamento inatteso (aprosdoketon), l'effetto comico. Per aprosdoketon (o fulmen in clausula) si intende la parola o espressione inaspettata, usata cioè in modo diverso dal consueto e in contrasto con la situazione creata, spesso in chiusura di componimento. Il meccanismo comico è descritto già da Aristot., rhet.1412a 18 ss. Sul procedimento retorico dell'aprosdoketon (o improvisum) vd. CELENTANO 1999.

16. KRUUSE 1941.

17. Vd. PICONE et all. 2003, p. 334.

18. BARTWICK 1959. La battuta a sorpresa usata da Marziale si configura come una sententia ex inopinato: vd. Quint. inst. 8, 5, 15.

19. La figura del bellus homo ricorre nella commedia, nella satira e nell'epigramma: vd. e.g. Catull. 24, 7; Hor. sat. 1, 2, 26-27; 6, 30 ss.; Ov. ars 3, 433 ss.; Gell. 6, 12, 5. Cf. FUSI 2006, pp. 405-406.

20. Evidente appare la struttura chiastica dei primi due versi: Cotile, bellus homo (v. 1) ... dic mihi, bellus homo (v.2), così come il parallelismo della struttura sintattica, caratterizzata da una serie di proposizioni relative, con l'anafora del relativo qui.

21. Cf. Theophr. char. 5, 6-7. Sul rapporto tra Marziale e Teofrasto, vd. RUSTEN 2002, p. 18.

22. Vd. RUSTEN 2002, p. 148; DIGGLE 2004, pp. 78; 222-223. Tutti i manoscritti, ivi compreso il PHerc. 1457 (I sec. d.C.), fanno seguire ai primi cinque paragrafi, in cui si descrive un uomo cerimonioso, una sezione di testo che sembra ritrarre un tipo umano diverso, che spende molto ostentando ricchezza ed affettazione. È possibile, quindi, che il capitolo che oggi leggiamo, sia nato dall'unione di due diversi caratteri, considerati in certo modo molto affini e perciò riuniti dal compilatore. Inoltre, il fatto che il bellus homo di Marziale condivida comportamenti e atteggiamenti solo con questo secondo carattere, potrebbe essere un ulteriore indizio a conferma della distinzione in due sezioni, una dedicata al piacente, l'altra al dandy. 
23. PASQUALI 1956, pp. IX-X.

24. USSHER 1960; EDMONDS 1961. Del resto, la caratterizzazione dei personaggi assume un rilievo particolare nella composizione di testi per la scena teatrale e all'analisi di Aristotele circa la tragedia (Aristot. poet. $15=1454 \mathrm{a} 16-\mathrm{b} 18$ ) deve essere corrisposta un'analoga indagine per i ruoli della commedia.

25. FORTENBAUGH 1984; TORRACA 1994, pp. XXIII-XXVI. Per una rassegna delle diverse posizioni degli studiosi in merito alla finalità dei Caratteri di Teofrasto e al loro genere letterario, vd. MATELLI 1989.

26. RUSTEN 2002, p. 19 e, soprattutto, GILL 1983.

27. Cf. TORRACA 1994, pp. XVIII-XXIV. Per Eraclide Pontico vd. Diog. Laert. 5, 88; per Satiro di Callati, vd. Athaen. 168c-d; per Aristone di Chio, vd. Phldm. vit. col. X, 10 ss. (= fr. 13 Wehrli). Anche Posidonio, filosofo stoico, parla della possibilità di impiegare a fini parenetici la descrizione dei caratteri, detta ethologia: vd. infra, n. 30.

28. Coppola 1945 , p. 52 ha tradotto quattro caratteri di Aristone di Chio ricavandoli dalla lettura del De vitiis di Filodemo (coll. XVI, 29 - XXIV, 27 Jensen = fr. 14 Wehrli).

29. Possiamo renderci conto della struttura tipica di queste descrizioni considerando la

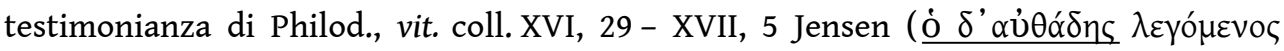

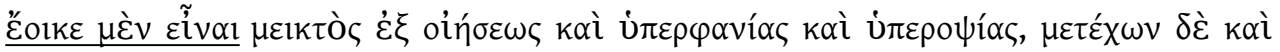

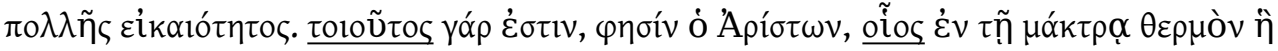

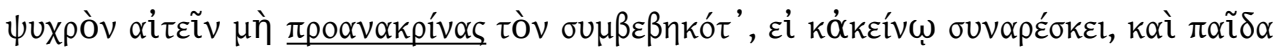

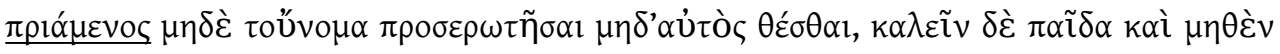

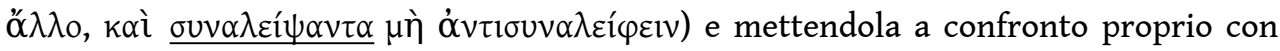
l'inizio del capitolo 5 dei Caratteri di Teofrasto dedicato al piacente (5, 1-2: $\dot{\eta} \delta \dot{\varepsilon}$

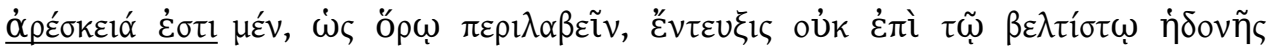

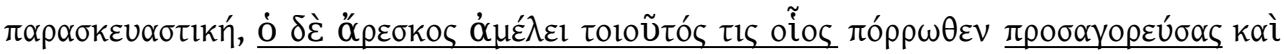

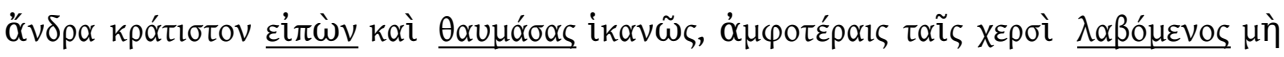

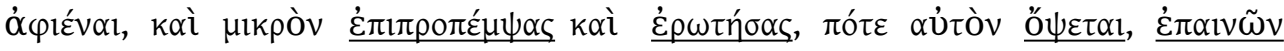

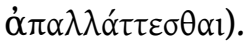

30. CALBOLI 1998, pp. 75-80.

31. Cic. top. 83: additur autem descriptio, quam $\chi \alpha \rho \alpha \kappa \tau \tilde{\eta} \rho \alpha$ Graeci vocant ... descriptio qualis sit avarus, qualis adsentator ceteraque eiusdem generis, in quibus et natura et vita describitur

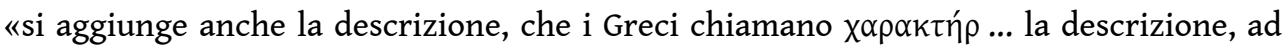
esempio: come è un avaro, come è un adulatore, e altre rappresentazioni di questo genere, nelle quali si descrive e la natura e la vita».

32. Cf. Quint. inst. 9, 2, 102 (per cui vd. BARABINO 1967, pp. 9-12).

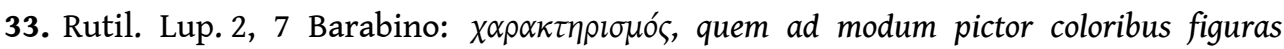
describit, sic orator hoc schemate aut vitia aut virtutes eorum de quibus loquitur, deformat "'characterismos: come il pittore dipinge le figure con i colori, così l'oratore rappresenta con questa figura retorica o i vizi o le virtù di coloro di cui parla». Sulla figura del characterismos, vd. DE TEMMERMAN 2010, pp. 29 ss.; BERARDI 2015.

34. Sen. epist. 95, 65-66: ait utilem futuram et descriptionem cuiusque virtutis; hanc Posidonius 'ethologian' vocat, quidam 'characterismon' appellant, signa cuiusque virtutis ac vitii et notas reddentem, quibus inter se similia discriminentur "dice che è utile altresì la definizione di ciascuna virtù; Posidonio la chiama ethologia, alcuni characterismos, 
poiché restituisce di ciascun vizio e ciascuna virtù i segni e le note distintive, per mezzo delle quali comportamenti simili possono essere distinti tra loro».

35. Vd. Aul.Gell. 12, 4, 1-2.

36. Vd. TORRACA 1994 , p. XXVIII. In vero l'osmosi tra i due diversi ambiti è stata preparata da Aristotele stesso il quale già notava la necessità da parte dell'oratore di conoscere i costumi e i comportamenti dei personaggi perché il discorso potesse risultare persuasivo. Un discorso è credibile nella misura in cui colui che parla si esprime con parole confacenti al suo ethos, determinato dalla condizione sociale, dall'età, dall'indole naturale, dall'educazione: Aristot. rhet.1408a 10-11; 1416a 16-23; vd. anche 1408a 24-31; 1356a 1-13.

37. Sui rapporti tra la letteratura ritrattistica, come quella dei Caratteri di Teofrasto, e l'esperienza della commedia nuova vd. FORTENBAUGH 1981; Hunter 1985, pp. 148-151; RUSTEN 2002, pp. 16-18. Questi rapporti assumono le forme della leggenda quando si parla di 'Menandro filosofo', allievo di Teofrasto, come in Diog. Laert. 5, 36 (per cui vd. GAISER 1967; MASSIONI 1998). Ancor più interessanti sono i rapporti tra la commedia nuova e la retorica che corrono lungo lo studio e l'imitazione dei caratteri; non a caso Quintiliano (inst. 10, 1, 27) raccomanda all'aspirante oratore di leggere poesie e commedie per prendere spunti utili alla caratterizzazione dei personaggi ed è significativo che il retore faccia riferimento a Teofrasto come alla fonte della dottrina.

38. Quint. inst. 6, 2, 17.

39. Svet. gramm. 4, 7; Quint. inst. 1, 9, 3.

40. Quint. inst. 1, 9, 3.

41. Sull'argomento vd. GRANATELLI 1995, pp.142-145 (a favore di ethologia); ROBINSON 1920; VACHER 1993, pp. 73-75 e infine BERARDI 2013 (contro).

42. Riguardo alle fonti progimnasmatiche, vd. Theon 70-73 Patillon (la simulazione del discorso è chiamata prosopopea alla maniera di Quint. inst. 3, 8, 49-54); Ps.Hermog. 9 Patillon; Aphthon. 11 Patillon; Nic. 63, 10 - 67, 15 Felten. Per uno studio complessivo dell'esercizio scolastico dell'etopea, vd. AMATO, SCHAMP 2005; KRAUS 2007.

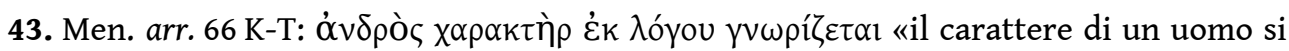
riconosce dal discorso».

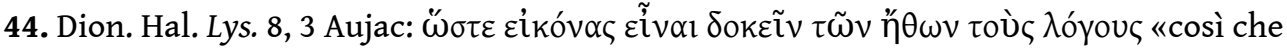
i discorsi sembrano essere immagine dei caratteri».

45. Cic. de orat. 3, 205: imitatio morum et vitae sive in personis sive sine illis. Poiché Cicerone parla di questa figura come una quasi dicendi virtus, siamo sicuri che essa corrisponda all'etopea, considerata da altri retori qualità elocutiva: vd. Dion. Hal. epist.Pomp. 3, 18; Lys. 8 Aujac.

46. Quint. inst. 9, 2, 58: imitatio morum alienorum, quae $\eta \dot{\theta o \pi}$ olía vel, ut alii malunt, $\mu i ́ \mu \eta \sigma \iota \varsigma$ dicitur, iam inter leniores adfectus numerari potest: est enim posita fere in eludendo. Sed

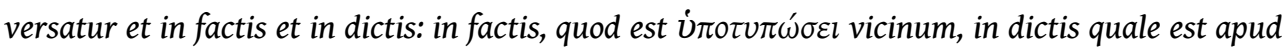
Terentium: "Aut ego nescibam quorsum tu ires?" (segue citazione tratta da Ter. eun. 155-157) «la rappresentazione dei caratteri altrui, che si chiama etopea o, come alcuni preferiscono, mimesi, può essere annoverara tra i sentimenti più leggeri: consiste infatti pressoché esclusivamente nel prendere in giro. Ma si esplica attraverso i fatti o le parole: attraverso i fatti, procedimento che è vicino all'ipotiposi, attraverso le parole, come si legge in Terenzio: 'o io non sapevo fin dove tu arrivassi'». 
47. Quint. inst. 9, 3, 99, laddove rifiuta la definizione di etopea e di characterismos data da Rutilio Lupo; per un'analisi di questi argomenti vd. BERARDI 2015.

48. Sull'equivalenza tra notatio ed etopea o characterismos, vd. le considerazioni di CALBOLI 1969, pp. 420-421.

49. Rhet. Her. 4, 63: notatio est cum alicuius natura certis describitur signis, quae, sicuti notae quaedam, naturae sunt adtributa; ut si velis non divitem, sed ostentatorem pecuniosi describere... «la descrizione del carattere si realizza quando la natura di un uomo è rappresentata con segni definiti che, come dei marchi, sono attribuiti alla natura, come ad esempio, se vuoi descrivere non un ricco, ma un ostentatore di ricchezze...» Vd. anche Rutil. Lup. 2, 7 Barabino.

50. Rhet. Her. 4, 65: huiusmodi notationes, quae describunt quod consentaneum sit unius cuiusque naturae... «descrizioni di questo genere, che rappresentano ciò che è consentaneo alla natura di ciascuno...»

51. DIGGLE 2004, pp. 9-10; i testi di riferimento, relativi principalmente ad Aristone di Chio, sono in WEHRLI 1968.

52. Sul contributo della descrizione particolareggiata alla visualizzazione del testo, vd. Demetr. eloc. 209-212. Il concetto è illustrato da RABAU 1995; MANIERI 1998, pp. 133-137; BERARDI 2012, pp. 33-39.

53. Rhet. Her. 4, 65: huiusmodes notationes ... totam enim naturam cuiuspiam ponunt ante oculos «descrizioni di questo genere ... pongono davanti agli occhi il carattere di qualcuno». L'espressione ante oculos ponere descrive l'effetto di evidenza visiva prodotto da meccanismi espressivi, come la metafora, la similitudine, la descrizione (o ipotiposi), capaci di suscitare nel lettore / ascoltatore l'impressione di una partecipazione diretta ai fatti raccontati. I fatti sono rappresentati, più che narrati, e al lettore pare di vedere le immagini di quanto descritto: Rhet. Her. 4, 51; 68; Cic. de orat. 3, 202; Quint. inst. 8, 3, 62-65; 9, 2, 40-44; Dion. Hal. Lys. 7 Aujac.

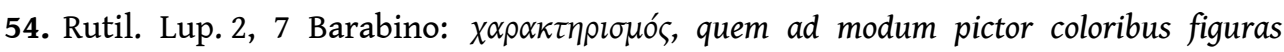
describit, sic orator hoc schemate aut vitia aut virtutes eorum de quibus loquitur, deformat. Così condensa la definizione il Carmen de figuris $(148=R L M$ p. 69 Halm): fit depictio, cum verbis ut imagine pingo «si realizza il ritratto, quando dipingo a parole come in un'immagine». Nella sua definizione Rutilio Lupo fa riferimento ad una lunga tradizione che pone a confronto l'arte della parola e quella del disegno relativamente alla rappresentazione dei sentimenti e dei comportamenti dell'uomo. Benché, infatti, la pittura sia svantaggiata nella raffigurazione dei caratteri umani, giacché è difficile rendere con la forma e con il colore ciò che non è materiale, esiste tuttavia la possibilità di rendere attraverso alcuni particolari (dello sguardo, dei lineamenti del volto) lo stato d'animo dei personaggi: cf. Xen. mem. 3, 10, 1-8; molti pittori vi sono riusciti e la letteratura ecfrastica ha colto questo aspetto: cf. e.g. Plin. nat.hist. 35, 69; Luc. Herod. 4-6; imag. 12; pisc. 38; Philostr. imag. proem. 3. Il biografo Plutarco, infine, elabora nella maniera forse più esauriente il rapporto tra le due arti circa la rappresentabilità dell'ethos: come i pittori selezionano i particolari del volto e del viso per rappresentare il carattere dell'individuo, così il biografo entra nell'anima riuscendo a disegnare il ritratto di ciascun uomo (Plut. Alex. 1, 3); egli riesce a riprodurre il carattere e la condotta del personaggio, mentre la pittura restituisce solo l'immagine visibile del corpo e del viso (Plut. Cimon. 1, 2). Su questi concetti vd. ZANGARA 2007, pp. 85 ss. 
55. LAUSBERG 1990, p. 406: la descrizione di carattere è classificata tra le figure retoriche dell'evidenza visiva.

56. Sul meccanismo di attivazione dell'evidenza, connesso alla rielaborazione di immagini depositate nella mente umana e attivate dalla fantasia, vd. BERARDI 2012, pp. 94-97 (tra le fonti antiche vd. Quint. inst. 6, 2, 29-34; 8, 3, 64-65).

57. Rhet. Her. 4, 65: huiusmodi notationes ... vehementer habent magnam delectationem "descrizioni di questo genere hanno grande piacevolezza».

58. Quint. inst. 9, 2, 58: imitatio morum alienorum... est enim posita fere in eludendo «la rappresentazione del carattere altrui ... consiste quasi esclusivamente nel canzonare».

59. Dion. Hal. Lys. 8; 10, 1-2; 13, 4 Aujac; su questi argomenti cf. BRUss 2013.

60. Rhet. Her. 4, 64: Quid ego quae deinde efficiat narrem? Eiusmodi est hominis natura ut quae singulis diebus efficiat gloria atque ostentatione ea vix annuo sermone enarrare possim «perché io dovrei narrare le cose che fa? La natura dell'uomo è tale che a stento potrei narrare con il discorso di un anno le cose che in singoli giorni egli porta a termine per gloria o ostentazione».

61. Il verbo narrare è equivalente del greco $\delta$ inүoũ $\mu \alpha$ l, che spesso è usato per indicare l'azione del descrivere. Infatti, nei progymnasmata l'ekphrasis è definita come una forma particolare di racconto, da cui si distingue per la virtù espressiva dell'evidenza: vd. soprattutto Nic. 68, 8 - 69, 3 Felten. Su questi argomenti cf. PATILLON 1997, pp. XXXIXXXIV. In questo senso è significativo che Marziale $(12,92,3)$ usi l'espressione narrare mores per indicare il ritratto del carattere.

62. Catull. 24, 7-8: "Qui? Non est homo bellus?" Inquies. Est; / sed bello huic neque servus est neque arca «'Perché? Non è un uomo piacente?' Dirai. Lo è; ma questo uomo elegante non ha né il servo né il patrimonio».

63. Hor. sat. 1, 2, 26-27; 6, 30 ss.

64. Vd. e.g. Mart. 1, 9; 2, 7; 12, 39; qualcuno ha individuato anche in Mart. 1, 96 i tratti tipici del bellus homo per l'ostentata eleganza del personaggio, ma l'aggettivo bellus non compare (vd. estellés GonZÁlez 2009). Per i ritratti tipologici, vd. per esempio la descrizione del cittadino elegante (urbanus) in Mart. 1, 41; o dell'ubriacone in Mart. 1, 87; 3, 82; o del ghiottone in Mart. 7, 20; o dell'uomo raffinato in Mart. 2, 29; 3, 12; 5, 61.

65. Mi riferisco soprattutto a Hor. epist. 1, 18 per cui Porfirione, il commentatore di Orazio, e gli scoliasti successivi hanno parlato di characterismos: Schol. ad Hor. epist. 1, 18, 5: Mire per characterismon, id est per imaginationem, describit hominem tristem et amarum «in modo mirabile, attraverso il characterismos, cioè l'immaginazione, rappresenta un uomo triste e severo».

66. Mart. 1, 9: Bellus homo et magnus vis idem, Cotta, videri: / sed qui bellus homo est, Cotta, pusillus homo est «vuoi essere, Cotta, un uomo elegante e nello stesso tempo grande: ma chi è un uomo elegante, Cotta, è un uomo piccolo».

67. Mart. 2, 7: Declamas belle, causas agis, Attice, belle, / historias bellas, carmina bella facis, / componis belle mimos, epigrammata belle, / bellus grammaticus, bellus es astrologus, / et belle cantas et saltas, Attice, belle, / bellus es arte lyrae, bellus es arte pilae. Nil bene cum facias, facias tamen omnia belle, / vis dicam quid sis? Magnus es ardalio «Attico, declami in modo elegante, discuti le cause in modo elegante, racconti storie eleganti, fai poesie eleganti, componi mimi eleganti, scrivi epigrammi eleganti, sei un grammatico elegante, sei un astrologo elegante, canti e balli in modo elegante, o Attico, sei elegante nell'arte della 
lira, sei elegante nel gioco della palla. Poiché non fai nulla bene, ma tuttavia fai tutto in modo elegante, vuoi che ti dica chi sei? Sei un faccendone». Si possono notare, in questo epigramma, il parallelismo sintattico della struttura, l'anafora in poliptoto di bellus, la battuta a sorpresa che viene a concludere una richiesta di definizione dopo una lunga descrizione delle attività dell'uomo.

68. Mart. 12, 39: Odi te quia bellus es, Sabelle. / Res est putida bellus et Sabellus. / Bellum denique malo quam Sabellus / Tabescas utinam, Sabelle, belle "ti odio perché sei un belloccio, Sabello. È una cosa fetida, il belloccio e anche Sabello. In breve, preferisco essere belloccio più che Sabello. Voglia il cielo che tu ti dissolvi bellamente, Sabello».

69. Sul contributo dell'anafora e della coordinazione per asindeto alla visualizzazione del testo, vd. Tib. fig. 29, 6; 40 Ballaira e Ps.Long. subl. 20, 1 (per cui cf. BERARDI 2012, pp. 32; 203).

70. La necessità che sia osservato un certo ordine nella descrizione dei fatti perché questo favorisce la visualizzazione del testo, è insita nel concetto di ekphrasis come 'discorso periegematico': cf. Theon 66, 7-8 Patillon; Ps.Hermog. 10, 1, 1-2 Patillon; Aphthon 12, 2, 1-2 Patillon. Sul significato di 'discorso periegematico', vd. Ioann. Sard. in Aphthon. progymn. RhG 15, 216, 14-16 Rabe; Ioann. Dox. proleg. ad Hermog. inv. RhG 14, 362, 23 - 363, 1 Rabe. L'ordine può essere: dall'alto in basso per persone, statue, etc.: Nic. 69, 12-17 Felten; prima, durante e dopo per fatti: Theon 68, 16-18 Patillon; dal centro alla periferia o viceversa per i luoghi: Aphthon. 12, 1 Patillon. Riguardo a questi argomenti, vd. DUBEL 1997; BERARDI 2010, pp. 41-42; 2012, pp. 195-198.

71. Vd. e.g. Plaut. Asin. 627; Iuv. 2, 40-42; Mart. 3, 12; 5, 61. Sul particolare dell'unguento e del profumo, vd. anche Mart. 2, 12; 6, 55. Questi tratti presentano il bellus homo come un personaggio effeminato: FUSI 2006, pp. 407-408.

72. Il processo di immaginazione letteraria (fantasia) consiste, infatti, nella rielaborazione di immagini depositate nella memoria e opportunamente attivate: vd. supra, n. 55.

73. Alludo al divertente epigramma di Mart. 5, 54: Extemporalis factus est meus rhetor: / Calpurnium non scripsit, et salutavit «è diventato un improvvisatore il mio retore: non ha scritto Calpurnio, eppure lo ha salutato». La canzonatura del retore, incapace persino di salutare perché ormai troppo abituato a leggere i suoi testi, avviene con dovizia di termini tecnici: l'aggettivo extemporalis è impiegato in accezione affine all'uso che ne fa la precettistica retorica per definire l'abilità dell'oratore nella composizione estemporanea del discorso (Quint. inst. 10, 7, 16; 18). Sul concetto di extemporalis ed extemporalis facilitas come capacità di improvvisazione, vd. CELENTANO 2010.

74. Sulla parodia nella retorica greca e latina, vd. celentano 1999. Tra le fonti, Quint. inst. 6, 3, 96-98; 9, 2, 34-35.

75. L'occhio attento di Marziale coglie molti di loro: vd. e.g. Mart. 2, 64; 3, 25; 5, 21; 5, 54; $5,56$.

76. Luc. praec.rhet. 11. Nel tono e nella forma espressiva del brano lucianeo ritroviamo molti tratti che distinguono il modello retorico della descrizione del carattere.

77. Echi di questo dibattito si ritrovano nell'Ars poetica di Orazio che dedica al problema della caratterizzazione dei personaggi, alla verosimiglianza dei costumi dei protagonisti e alla vivezza espressiva del testo molte considerazioni: vd. Hor. ars 114-130; 153-157; 180-182; 360-364. 
78. È del resto noto come i procedimenti di visualizzazione testuale determinino effetti di immediatezza visiva e di naturalezza nella rappresentazione per cui l'evidenza (enargeia) è considerata mezzo espressivo tipico del realismo letterario: vd. ZANKER 1987, pp. 39-50. Questo concetto era conosciuto agli antichi: vd. Dion. Hal. Lys. 13, 5 Aujac; Quint. inst. 8, 3, 70-71.

79. Vd. supra, n. 53.

80. Vd. IZAAC 1969, p. 17. La sensibilità per le qualità di evidenza visiva, che si evince dallo studio della ritrattistica di Marziale, ben si colloca nel generale apprezzamento per le doti di scrittura ecfrastica, che emerge dalle recenti indagini condotte da MORETTI 2010 sui libri XIII e XIV degli Xenia e degli Apophoreta.

81. È dei retori il precetto secondo cui la perizia artistica non deve riconoscersi, ma celarsi in una simulata spontaneità della scrittura: vd. Quint. inst. 12, 9, 5-6. Dove c'è troppa ostentazione d'arte, c'è senso di artificio, simulazione: Quint. inst.9, 3, 102. L'eccessivo apparato retorico produce affettazione e questa, a sua volta, inficia l'imitazione della vita reale: Quint. inst. 2, 10, 11.

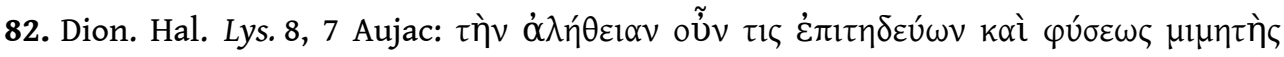

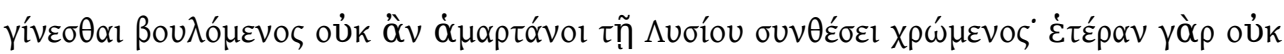

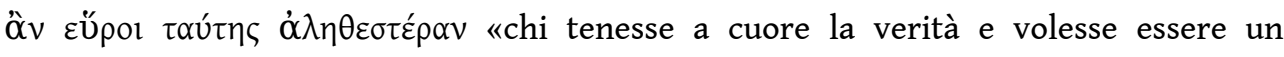
imitatore della natura, non sbaglierebbe a servirsi dello stile di Lisia: non troverebbe infatti nessuno stile più veritiero di questo». Il giudizio è condiviso anche da Quint. inst. 3, 8, 51: ideoque Lysias optime videtur in iis quae scribebat indoctis servasse veritatis fidem «e perciò sembra anche agli ignoranti che Lisia abbia conservato in maniera eccezionale la fedeltà alla verità nelle orazioni che ha scritto». Sulla etopea come qualità caratteristica dei discorsi di Lisia, vd. supra, n. 60.

\section{RIASSUNTI}

Gli artifici della retorica sembrano lontani dal realismo della poesia di Marziale. Al contrario, un'attenta analisi degli epigrammi mostra come il meccanismo comico e la vivezza descrittiva che li caratterizzano siano debitori di molti procedimenti espressivi illustrati dai manuali tecnici. Infatti, lo studio del mordace ritratto di un dandy (il bellus homo di 3,63) rivela in filigrana la rielaborazione di un esercizio di scrittura diffuso nelle scuole. Ne scaturisce una nuova possibile chiave di lettura del brillante epigramma.

Il semble que les artifices de la rhétorique soient étrangers au réalisme de la poésie de Martial. Au contraire, l'analyse des épigrammes montre comment le mécanisme comique et la vivacité de la description résultent des procédés d'écriture qui ont été illustrés par les artes rhetoricae. En fait, l'étude d'un tableau qui représente un dandy (bellus homo) révèle une élaboration textuelle très habile, qui joue sur la structure d'un exercice scolaire, la description des caractères (éthopée). Cela permet de donner à l'épigramme une nouvelle interprétation.

It seems likely that Martial intended to write a realistic poetry far from rhetorical artificialities. On the contrary, by analyzing the epigrams, one can show how the comic mechanisms and the 
vivid descriptions are the result of the rules illustrated by the artes rhetoricae. The depiction picture of a dandy (bellus homo) shows a very clever textual elaboration based upon a school exercise, the ethopoiea. This may shed a new light on the meaning of this epigram.

\section{INDICE}

nomsmotscles Aphthonius, Apulée, Ariston de Chios, Aristote, Athénée, Aulu-Gelle, Basile de Césarée, Catulle, Cicéron, Démétrios de Phalère, Denys d'Halicarnasse, Diogène Laërce, Ennius, Héraclide du Pont, Pseudo-Hermogène, Horace, Ioannis Doxapatri, Jean (évêque de Sarde), Juvénal, Pseudo-Longin, Lucien de Samosate, Lucilius (Caius), Martial, Ménandre, Nicolas de Myre, Ovide, Philodème, Philostrate de Lemnos, Plaute, Pline l'Ancien, Plutarque, Priscien, Quintilien, Rutilius Lupus (Publius), Salluste, Satyros, Sénèque, Suétone, Tacite, Térence, Théon (Aelius), Théophraste, Tibulle, Xénophon

Keywords : bellus homo, description of characters, epigramm, ethopoeia, hypotyposis, rhetoric exercises, progymnasmata, rhetoric, picture

Mots-clés : bellus homo, description de caractères, épigramme, éthopée, évidence, exercices d'écriture, progymnasma, rhétorique, tableau

oeuvrecitee Rhétorique à Herennius

\section{AUTORI \\ FRANCESCO BERARDI}

Università degli Studi G. d'Annunzio Chieti e Pescar, DiLASS 